\title{
Sertraline Hydrochloride
}

National Cancer Institute

\section{Source}

National Cancer Institute. Sertraline Hydrochloride. NCI Thesaurus. Code C29451.

The hydrochloride salt of sertraline, a synthetic derivative of naphthalenamine with antiserotoninergic and anti-depressant properties. Sertraline appears to selectively inhibit the neuronal uptake of serotonin, raising serotonin levels in the CNS. 